\title{
Predictores del comportamiento de automanejo en personas con enfermedades crónicas de Tamaulipas
}

\section{Self-management behavior predictors among persons with chronic diseases in Tamaulipas}

\section{Preditores do comportamento de automanejo em pessoas com doenças crônicas de Tamaulipas}

\author{
R.C. León-Hernández ${ }^{\mathrm{a} 1}$, M.I. Peñarrieta-de Córdova ${ }^{\mathrm{b} 1}$,T. Gutiérrez-Gómez ${ }^{\mathrm{c} 1}$, \\ O. Banda-González ${ }^{\mathrm{d} 2}$, F. Flores-Barrios ${ }^{\mathrm{e} 1}$, M.C. Rivera ${ }^{3}$ \\ ORCID: \\ a $0000-0001-5780-4382$ \\ b $0000-0002-9293-4024$ \\ c $0000-0001-5522-4237$ \\ ${ }^{\mathrm{d}} 0000-0003-3540-6716$ \\ e 0000-0003-0340-3203 \\ ${ }^{1}$ Facultad de Enfermería Tampico. Universidad Autónoma de Tamaulipas \\ ${ }^{2}$ Facultad de Enfermería Ciudad Victoria. Universidad Autónoma de Tamaulipas \\ ${ }^{3}$ Jurisdicción Sanitaria No.2 Tampico. Secretaría de Salud Tamaulipas
}

Recibido: 24 septiembre 2018

Aceptado: 31 enero 2019

\section{Resumen}

Introducción: Dado el carácter prioritario de atención a las enfermedades no transmisibles, la Organización Mundial de la Salud propuso el Modelo de Cuidados Crónicos. En este modelo incluye el automanejo como un elemento principal en el combate de dichas enfermedades.

Objetivo:identificar las variables predictoras del comportamiento de automanejo en usuarios con Diabetes Mellitus, Hipertensión Arterial y Obesidad.

Métodos: La muestra no probabilística e intencional fue de 386 usuarios de la Secretaría de Salud de Tamaulipas (Ciudad Victoria y Tampico). El diseño de estudio fue no experimental, transversal.

Resultados: casi la mitad (42.2\%) de los participantes con ECNT refieren síntomas de trastorno depresivo en el continuo de leve a grave, la categoría que obtuvo mayor porcentaje de IMC fue sobrepeso con 
33.2\% y más de un tercio de la población (36.8\%) percibe algún grado de disfunción familiar. Así también se aplicó un análisis de Regresión Lineal Múltiple con el método de pasos, el modelo indica que fueron siete predictores del comportamiento de Automanejo (varianza explicada $R^{2}=.325$ ); la variable autoeficacia fue el más importante $(\beta=38)$ y el apoyo social percibido el predictor con carga más baja $(\beta=.10)$.

Conclusiones: estos predictores representan elementos importantes para ampliar el conocimiento y entender el comportamiento del automanejo, área que es promovida en el modelo de atención a crónicos de la Organización Mundial de la Salud y en la actualidad es liderada por el área de enfermería.

Palabras clave: Automanejo; Diabetes Mellitus; Hipertensión; México.

\section{Abstract}

Introduction: Considering the priority regarding the attention of non transmissible diseases, the World Health Organization proposed the Chronic Care Model which, by encompassing self-management as one main element, is intended to address these diseases.

Objective: To identify predictor variables related to behavior on self-management among patients with Diabetes Mellitus, Hypertension, and Obesity.

Methods: The intentional and non probabilistic simple included 386 patients from the Health Secretariat in Tamaulipas, Mexico (Ciudad Victoria and Tampico). The study design was non experimental and transversal.

Results: About half (42.2\%) of the participants with Chronic Non Transmissible diseases referred having depressive disorder ranging from mild to severe. Regarding the BMI, 33.2\% showed overweight. Moreover, $36.8 \%$ perceived some degree of family dysfunction. A Multiple Linear Regression was calculated. The model indicated that there were seven predictors of the behavior on self-management (explained variance $\left.R^{2}=.325\right)$; being self-efficacy the most important $(\beta=38)$, and perceived social support the lowest in load $(\beta=.10)$. Conclusions: These predictors represent important elements to consider when building on the knowledge and understanding the behavior related to self-management, an area which is highlighted in the Chronic Care Model of the World Health Organization, particularly within nursing. Keywords: Self-management; diabetes mellitus; hypertension; Mexico.

\section{Resumo}

Introdução: Dado o caráter prioritário de atenção às doenças não transferíveis, a Organização Mundial da Saúde propôs o Modelo de Cuidados Crônicos. Neste modelo inclui-se o automanejo como um elemento principal no combate de tais doenças.

Objetivo: identificar as variáveis preditoras do comportamento de automanejo em usuários com Diabetes Mellitus, Hipertensão Arterial e Obesidade.

Métodos: A amostra não probabilística e intencional foi de 386 usuários da Secretaría de Salud de Tamaulipas (Ciudad Victoria e Tampico). O desenho de estudo foi não experimental, transversal.

Resultados: quase a metade (42.2\%) dos participantes com ECNT referem sintomas de transtorno depressivo no continuo de leve a grave, a categoria que obteve maior porcentagem de IMC foi sobrepeso com $33.2 \%$ e mais de um terço da população $(36.8 \%)$ percebe algum grau de disfunção familiar. Assim também se aplicou uma análise de Regressão Linear Múltipla com o método de passos, o modelo indica que foram sete preditores do comportamento de Automanejo (variância explicada $R^{2}=.325$ ); a variável autoeficácia foi a mais importante $(\beta=38)$ no apoio social percebido e o preditor com carga mais baixa $(\beta=.10)$.

Conclusões: estes preditores representam elementos importantes para ampliar o conhecimento e entender o comportamento de automanejo, área que é promovida no modelo de atenção a crônicos da Organização Mundial da Saúde e na atualidade é liderada pela área de enfermagem.

Palavras chave: Automanejo; Diabetes Mellitus; Hipertensão; México. 


\section{Introducción}

Las Enfermedades Crónicas No Transmisibles (ECNT) como Diabetes Mellitus (DM), Enfermedades Cardiovasculares, Cáncer y Obesidad, entre otras, representan un problema de salud a nivel mundial. La Organización Panamericana de la Salud destaca que del total de la población que vive con una ECNT, únicamente la mitad está diagnosticada y sólo una cuarta parte recibe atención médica. Aún más preocupante es el hecho de que una de cada diez personas con estas enfermedades es tratada con éxito ${ }^{1}$.

Está documentado que dichas enfermedades representan la principal causa de muerte a nivel mundial con 38 millones de defunciones, 40\% de ellas se registraron en personas menores de 70 años $^{2}$. En la región de las Américas, estas enfermedades fueron causa de 4.5 millones de muertes ${ }^{3}$.

Datos más específicos indican que la prevalencia mundial de DM se estima en $8.5 \%$, se acentúa en países de ingresos bajos y medios ${ }^{4}$. En el caso de México, la Encuesta Nacional de Salud y Nutrición de Medio Camino (2016), reportó las siguientes prevalencias para ECNT con diagnóstico previo en adultos: DM 9.4\%, hipertensión arterial (HTA) 25.5\%, sobrepeso y obesidad combinados 72.5\%. Cabe señalar que casi la mitad de las participantes con HTA (40\%) desconocía su diagnóstico 5 . Además, se ha documentado un efecto mediador entre IMC alto, presión arterial sistólica y glucosa en sangre ${ }^{6}$.

El costo de estas enfermedades es alto, un estimado del impacto económico indica que en México se invierten entre el 73 y $87 \%$ del gasto programable para salud en DM asociada con sobrepeso y obesidad ${ }^{7}$. A nivel mundial, el costo proyectado para las ECNT en el 2030, será de 458 miles de millones de dólares $^{1}$, y las pérdidas económicas para los países de ingresos bajos y medios, se estima en siete billones de dólares en el periodo 2011-2025².

Dado el carácter prioritario de atención a las ECNT, la Organización Mundial de la Salud (OMS) propuso el Modelo de Cuidados Crónicos, que se enfoca en promover la relación entre un paciente activado con un equipo de profesionales de la salud preparados y proactivos. En este modelo se plantearon seis componentes, entre los cuales se encuentra el Apoyo al Automanejo, que es definido como el suministro sistemático de educación e intervenciones de apoyo por parte de los profesionales de la salud, con el fin de incrementar las habilidades y la confianza de los pacientes en el manejo de sus problemas de salud ${ }^{1}$.

El concepto de automanejo, como reseñan Lorig y Holman ${ }^{8}$, tiene sus orígenes en la teoría del aprendizaje social de Bandura y en la aportación del concepto de autoeficacia realizado como parte del desarrollo de su teoría. Grady y Gough lo definen como la atención diaria, por parte de las propias personas que cursan con alguna enfermedad crónica, es decir aquellas tareas que las personas sanas realizan en el hogar para prevenir enfermedades, en lugar de tan solo atender una enfermedad existente ${ }^{\text {. }}$ Por su parte, Lawn, Battersby, Harvey, Pols y Ackland, lo definen como la forma de evitar las complicaciones innecesarias, lo que maximiza la calidad de vida con el aumento de apoyo informal y formal. Estos últimos autores diseñaron un modelo de automanejo que integra la capacidad de la persona para comprender la naturaleza de su enfermedad, de gestionar y organizar su acceso a elementos importantes de su cuidado y adoptar formas de vida que promuevan la salud ${ }^{10}$.

Una conceptualización más realizada en un análisis dimensional de literatura científica, refiere que el automanejo se compone de cinco dimensiones: recursos, conocimientos, adherencia, participación activa y toma de decisiones informadas en referencia a algún padecimiento ${ }^{11}$.

Los estudios sobre el comportamiento de automanejo en personas con DM han documentado que se asocia con factores socio-demográficos, como edad, género, nivel educativo, estado civil y país de nacimiento, además, se relaciona con factores clínicos como indicadores de salud general y factores psicológicos como depresión, confianza, conocimientos y educación en salud ${ }^{12}$. También, en un estudio realizado en la India se reportaron correlaciones entre automanejo y problemas de salud mental, como ansiedad, depresión, disfunción social y síntomas somáticos ${ }^{13}$.

Estudios recientes han documentado variables predictoras de automanejo en diferentes poblaciones: un estudio realizado en Malasia reportó que la creencia en la efectividad de tratamiento en personas con DM, el apoyo familiar y la autoeficacia funcionan como predictores de automanejo ${ }^{14}$, en Australia los 
predictores de automanejo deficiente fueron; edad menor a 60 años, tener estudios superiores de educación secundaria, depresión y bajo conocimiento en manejo de $\mathrm{DM}^{12}$. En Inglaterra se documentó en personas con escaso control de DM, que la percepción de enfermedad y autoeficacia son los predictores más importantes de automanejo ${ }^{15}$. Finalmente, en una muestra iraní se reportó que la autoeficacia, las creencias en la efectividad del tratamiento y el soporte social predicen el automanejo en $\mathrm{DM}^{16}$.

En México se han realizado estudios para validar instrumentos ${ }^{17} \mathrm{y}$ evaluar el comportamiento de automanejo en personas con ECNT (generalmente deficiente), y su relación estadísticamente significativa con percepción de funcionalidad familiar ${ }^{18,19}$. Asimismo se documentó que la interacción entre automanejo e intensificación del tratamiento contribuyen a disminuir el nivel glucémico en personas con DM, dicha asociación se presentó sólo en la interacción de las dos variables, ya que el automanejo por sí sólo no contribuye en el control glucémico ${ }^{20}$.

Se documentó también la efectividad del programa de automanejo "tomando control de su salud" en personas con DM e HTA de Tampico-Tamaulipas, cuyos resultados muestran incrementos significativos en el comportamiento de automanejo en grupo intervenido comparado con un grupo control ${ }^{21}$. De igual forma, se evaluó el "proyecto vida" que incluye el apoyo al automanejo como parte del modelo de atención a crónicos de la OMS, cuyos resultados muestran un incremento de casi el 10\% de participantes con control glucémico 22 .

Como se puede evidenciar, el comportamiento de automanejo puede ser complejo, debido a la interacción con distintas variables que intervienen en el proceso de desarrollo del mismo. Analizar la interrelación entre las variables es de suma importancia, ya que permitirá entender, prever y potenciar el automanejo en pacientes con ECNT. Por lo anterior, el objetivo de este trabajo es identificar variables predictoras del comportamiento de automanejo en usuarios con DM, HTA y Obesidad de Tamaulipas.

\section{Material y métodos}

Muestra no probabilística, conformada por 386 usuarios de centros de salud de la Secretaría de Salud de Tamaulipas, Jurisdicciones: 1) Ciudad Victoria $(n=164)$ y 2) Tampico $(n=222)$, con diagnóstico inicial de Diabetes Mellitus, Hipertensión Arterial y/o Obesidad. El muestreo intencional se realizó bajo las condiciones de la medición basal de un proyecto mayor que tiene como propósito hacer frente al problema de salud que representan las Enfermedades Crónicas en las personas de Latinoamérica, así como promover el automanejo como un componente importante en la atención de su salud. Dicho proyecto es liderado por la Facultad de Enfermería Tampico de la Universidad Autónoma de Tamaulipas (FET-UAT) y se apega a las condiciones del programa Tomando Control de su Salud desarrollado por la Universidad de Stanford.

Los criterios de inclusión para el presente estudio fueron personas mayores de edad, que tenían uno y/o comorbilidad con los tres diagnósticos antes mencionados con un mínimo de seis meses de detección y que firmaran el consentimiento informado para participar en la investigación. Se excluyeron a las personas que presentaron algún padecimiento médico o psicológico que interfiriera con los fines de la investigación. El levantamiento de datos se realizó de febrero a julio de 2018, con un diseño de estudio no experimental y transversal.

La investigación implicó el uso de diversos instrumentos para medir las variables consideradas. Para variables independientes en primer lugar se evalúo el Índice de masa corporal (IMC), se obtuvo mediante el peso y la talla (IMC=peso/talla ${ }^{2}$ ), que fueron recolectados previo a la aplicación de cuestionarios y se clasificaron de acuerdo a los criterios de la OMS. La edad de los participantes, el diagnóstico y los años con este, los años de escolaridad, se obtuvieron mediante la cédula de datos sociodemográficos.

El funcionamiento familiar se evaluó con la escala de percepción APGAR familiar. La versión en español tiene un alfa de Cronbach de 0.80 y la validez de criterio oscila entre 0.71 y 0.83 . El instrumento consta de cinco preguntas en una escala tipo Likert de 0 a 4 . La interpretación del instrumento es, a mayor 
puntaje mejor percepción de funcionalidad familiar en cuatro categorías diferentes: funcionamiento normal, disfunción leve, moderada y severa ${ }^{23}$.

El apoyo social se midió a través del Instrumento Duke-UNC-11. Consta de 11 ítems con una escala de respuesta tipo Likert de 1 a 5 . Se califica realizando la sumatoria de todos los ítems para obtener un puntaje global de apoyo social o al menos considerar dos áreas: apoyo confidencial (ítems 1, 4, 6, 7, 8 y 10) y apoyo afectivo (ítems 2, 3, 5, 9 y 11), su interpretación es puntajes menores expresan escaso apoyo. Fue validado en México con un alpha de Cronbach de 0.92 y una validez de constructo que explica el $58.6 \%$ de la varianza con un solo factor ${ }^{24}$.

Actividad física se evaluó con la escala de Comportamiento de ejercicio que tiene confiabilidad test-retest de 0.72 , se compone de seis reactivos que evalúan la frecuencia de ejercicio (en minutos) realizado por semana, con cinco opciones de respuesta que van de $0=$ ninguno hasta $4=$ más de 3 horas. Se califica sumando el total de ítems, o bien con los 5 ítems de ejercicio aeróbico ( 2 a 6) e interpretando el reactivo 1 de fortalecimiento de forma independiente. Para el presente trabajo se realizó la sumatoria del total de ítems ${ }^{25}$.

Para evaluar sintomatología de trastorno depresivo se utilizó el Personal Health Questionnaire Depression Scale (PHQ-8), que está integrado por ocho preguntas, cada una con cuatro alternativas de respuesta (de menor a mayor frecuencia). Cada ítem tiene un valor que va de 0 a 3 puntos, su sumatoria genera una puntuación global que va de 0 a 24 puntos totales. La puntuación global puede interpretarse por categorías que van desde ausencia hasta síntomas graves ${ }^{26}$. En México se obtuvo un alpha de Cronbach de 0.81 y validez de criterio de $0.75^{27}$.

Autoeficacia para el manejo de enfermedades crónicas es una escala que como su nombre lo indica evalúa autoeficacia. Está integrada por seis preguntas con una escala de calificación continua de 1 a 10, la consistencia interna es de 0.91. Los puntajes más altos indican mayor autoeficacia ${ }^{28}$.

La variable dependiente: Automanejo, fue medida a través del Instrumento Partners in Health Scale $(\text { PHS })^{29}$, validado en contexto mexicano por Peñarrieta-de Córdova et al, en 2014. Este instrumento permite valorar el comportamiento en forma global y en tres dimensiones: 1) conocimiento sobre su salud y enfermedad, 2) manejo del impacto físico, emocional y social derivado de su enfermedad y 3 ) la dimensión de adherencia entendida no sólo en adherencia al tratamiento médico sino también en la comunicación con el médico y proveedor de salud. El instrumento está formado por 12 ítems con una escala de calificación continua que va de 0 a 8 y un alpha de Cronbach de 0.88 , su interpretación es a mayor puntaje mejor automanejo ${ }^{17}$.

Procedimiento. Una vez obtenidos los permisos de las instituciones correspondientes, se capacitaron a docentes y estudiantes de pregrado y posgrado de la Facultad de Enfermería de la UAT, sedes Tampico-Madero y Ciudad Victoria, así también a personal de enfermería de las jurisdicciones 1 y 2 de la Secretaría de Salud de Tamaulipas. La convocatoria para participar en el estudio lo llevaron a cabo enfermeras de 15 Centros de Salud de los dos municipios antes mencionados, las personas con ECNT que decidieron participar, lo hicieron de forma voluntaria y firmaron la carta de consentimiento informado, la toma de las medidas antropométricas y la aplicación de la encuesta se realizó en aproximadamente 30 minutos, por persona. El proyecto fue aprobado por el comité de ética y el de investigación de la FET-UAT y de la Secretaría de Salud de Tamaulipas. Los cuidados éticos se apegaron a la Ley general de Salud en Materia de Investigación. Los datos fueron procesados a través del Paquete Estadístico para las Ciencias Sociales (SPSS por sus siglas en inglés), versión 23 para Windows.

\section{Resultados}

La muestra estuvo formada por personas de ambos sexos (mujeres $n=361$ y hombres $n=25$ ), con diagnóstico de Diabetes Mellitus Tipo 2 ( $n=191)$, Hipertensión Arterial $(n=170)$ y Obesidad $(n=25)$, que fue detectado entre 10 meses y 39 años atrás (=9.74 \pm 7.87$)$. Los participantes fueron usuarios de 
centros de salud de la Secretaría de Salud de Tamaulipas; Jurisdicción 2 de Tampico (n=222) y Jurisdicción 1 Ciudad Victoria $(n=164)$, con un rango de edad de 20 a 87 años $(=55.8 \pm 11.9)$.

En la Tabla 1 se destaca que casi la mitad (42.2\%) de los participantes con ECNT refieren síntomas de trastorno depresivo en el continuo de leve a grave. También, destaca que el resto de la muestra no reporta esta problemática. Con referencia al índice de masa corporal, la categoría que obtuvo mayor porcentaje de acuerdo a la clasificación de la OMS, fue sobrepeso con 33.2\%, cabe enfatizar que en esta tabla se presentan los porcentajes de IMC en el total de la muestra y no sólo en el grupo con el diagnóstico inicial de obesidad. Finalmente, más de un tercio de la población (36.8\%) percibe algún grado de disfunción familiar.

Tabla 1. Distribución porcentual en variables categóricas. Tamaulipas 2016

\begin{tabular}{lcc}
\hline \multicolumn{3}{c}{ Sintomatología de trastorno depresivo (n=386) } \\
\hline & $\mathbf{f}$ & $\%$ \\
\hline Ausencia de Síntomas & 223 & 57.8 \\
\hline Síntomas Leves & 106 & 27.5 \\
\hline Síntomas Moderados & 42 & 10.9 \\
\hline Síntomas Severos & 10 & 2.6 \\
\hline Síntomas Graves & 5 & 1.3 \\
\hline IMC. Categorías OMS (n=368) & 1 & 0.3 \\
\hline Bajo Peso (<18.5) & 53 & 14.4 \\
\hline Peso Normal (18.5-24.9) & 128 & 34.8 \\
\hline Sobrepeso (25-29.9) & 114 & 31.0 \\
\hline Obesidad 1 (30-34.9) & 41 & 11.1 \\
\hline Obesidad 2 (35-39.9) & 31 & 8.4 \\
\hline Obesidad 3 (>40) & & \\
\hline Funcionalidad Familiar (n=386) & 244 & 63.2 \\
\hline Funcionalidad Normal & 71 & 18.4 \\
\hline Disfunción Leve & 36 & 9.3 \\
\hline Disfunción Moderada & 35 & \\
\hline Disfunción Severa & & \\
\hline
\end{tabular}

En la Tabla 2 se presenta el valor de las medias y desviación estándar de las restantes variables de estudio, cabe señalar que todas a excepción de "Ejercicio", presentaron una distribución normal con base en los indicadores de asimetría $( \pm 1.5)$ y curtosis $( \pm 2)$. Es importante resaltar que la variable ejercicio fue recodificada con base a las indicaciones de los autores del instrumento original, motivo por el cual pudo presentar una distribución anormal. Por lo anterior, debido al interés de los investigadores se incluyó dicha variable en el análisis posterior.

Tabla 2. Media y desviación estándar variables numéricas. Tamaulipas, 2016

\begin{tabular}{lrrrr}
\hline & Mínimo & Máximo & \multicolumn{1}{c}{ Media } & DesvEst. \\
\hline Automanejo global & 11 & 96 & 73.59 & 16.90 \\
\hline Autoeficacia & 1 & 10 & 7.31 & 2.23 \\
\hline Años de estudio & 0 & 27 & 6.69 & 3.9 \\
\hline Apoyo Familiar & 11 & 55 & 41.57 & 12.66 \\
\hline Ejercicio en minutos & 0 & 630 & 68.43 & 94.59 \\
\hline
\end{tabular}

Con el propósito de explorar la capacidad predictiva de las variables independientes: edad, IMC, años de estudio, tiempo de detección del diagnóstico, sintomatología de trastorno depresivo, funcionalidad 
familiar, autoeficacia, apoyo familiar y ejercicio, sobre la variable dependiente "automanejo”, se aplicó un análisis de Regresión Lineal Múltiple con el método de pasos. El análisis concluyó en el modelo 7, el ANOVA (F25.65, p<.001) indica que existe una relación lineal significativa entre siete variables independientes con la dependiente.

Como se puede observar, la variable autoeficacia fue el predictor más importante $(\beta=.38)$ y el apoyo social percibido el predictor con carga más baja $(\beta=.10)$. Cabe destacar que sintomatología de depresión presenta una carga negativa $(\beta=-.13)$, que se explica con la forma de calificación del instrumento y se interpreta que a mayor depresión menor automanejo. La varianza explicada para Automanejo como efecto de las independientes es $\mathrm{R}^{2}$ ajustada=.325, por lo que se deja sin explicar más de dos tercios del porcentaje de la misma (67.5\%). Otro aspecto relevante del modelo fue que se excluyeron las variables IMC y edad.

Tabla 3. Variables predictoras del comportamiento de automanejo Tamaulipas (análisis de Regresión Lineal Múltiple)

\begin{tabular}{|c|c|c|c|c|c|c|}
\hline & $B(S E)$ & IC95\% & $\boldsymbol{\beta}$ & $\boldsymbol{T}$ & Sig & $\begin{array}{l}\boldsymbol{R}^{2} \\
\text { ajustada }\end{array}$ \\
\hline Autoeficacia & $2.83(0.34)$ & $2.15-3.51$ & .38 & 8.21 & $<.001$ & \multirow{7}{*}{$\begin{array}{l}.325 \text { (13.81 } \\
\text { error estimado) }\end{array}$} \\
\hline Funcionalidad familiar & $2.28(0.88)$ & $0.54-4.02$ & .13 & 2.58 & $<.05$ & \\
\hline Ejercicio en minutos & $0.24(.008)$ & $.008-.04$ & .13 & 2.94 & $<.05$ & \\
\hline Sintomatología Depresión & $-2.54(0.88)$ & $4.26-0.81$ & -.13 & -2.89 & $<.05$ & \\
\hline Años con diagnóstico & $0.26(.09)$ & $.07-.44$ & .12 & 2.75 & $<.05$ & \\
\hline Años de estudio & $0.42(.20)$ & $0.39-.81$ & .10 & 2.17 & $<.05$ & \\
\hline Apoyo social percibido & $0.14(.07)$ & $.002-.28$ & .10 & 2.0 & $<.05$ & \\
\hline
\end{tabular}

\section{Discusión}

Entre los resultados en el total de la muestra se encontró (conforme a los 3 diagnósticos iniciales: DM, HTA y Obesidad) un alto porcentaje de participantes que presentan sobrepeso y obesidad (81.3\%), que supera casi un $10 \%$ de lo reportado a nivel nacional ${ }^{5}$. También, destaca que casi un $20 \%$ de la muestra se clasifica en obesidad 2 y 3 (mórbida), lo cual los ubica en alto riego de salud. Si bien el IMC no figura como una variable predictora de automanejo, se sugiere investigar la relación inversa, es decir, explorar la ubicación de automanejo como predictora del IMC en futuras investigaciones.

El resultado de mayor relevancia muestra que el comportamiento de automanejo es complejo, de las nueve variables independientes consideradas en el análisis de regresión, únicamente siete entraron en el modelo y explican sólo un tercio de la varianza (32.5\%). Por lo anterior, se infiere que el automanejo puede ser pronosticado por otras variables que no se contemplaron en el presente estudio. Cabe mencionar que este porcentaje de varianza es mayor a los estudios realizados en Malasia, en el que se encontraron tres predictores (13\%) y Australia con cuatro predictores (17\%), sin embargo, está por abajo del estudio realizado en Irán en el que se reportó $89 \%$ de varianza explicada mediante tres variables predictoras $^{12,14,16}$.

Con respecto al valor predictivo, la variable autoeficacia mostró la $\beta$ más alta (.385), es decir, esta variable es la que mejor predice el comportamiento de automanejo. Resultados similares se detectaron en los modelos predictivos de automanejo desarrollados en Malasia, Inglaterra e Irán ${ }^{14-16}$. Cabe resaltar que aunque la diferencia cultural en los países antes señalados es innegable, la relación causa-efecto entre autoeficacia y automanejo es la misma en dichos contextos, lo anterior tiene un sustento teórico importante, ya que el comportamiento del automanejo tiene como antecedente la teoría del aprendizaje social y el concepto de autoeficacia de Bandura ${ }^{8}$, por lo anterior esta asociación será una directríz en el desarrollo de futuros modelos explicativos de la variable dependiente. 
En referencia a las variables del modelo de regresión, se ha documentado que depresión, ejercicio, escolaridad y años con el diagnóstico también aparecen como predictores de automanejo en diferentes poblaciones $^{12,14,15}$.

Uno de los aportes del presente estudio fue evidenciar la función predictora de las variables: percepción de funcionalidad familiar y apoyo social, en el comportamiento de automanejo. Si bien ya se habían documentado correlaciones en estudios antecedentes ${ }^{12,13,18,19}$, ninguno de ellos había explorado la capacidad predictiva de las variables antes señaladas, que evidencian la importancia de las relaciones socio-familiares en el automanejo de personas que cursan con algún padecimiento crónico como DM, HTA y/o obesidad.

Finalmente, el resultado en la variable edad (excluida del modelo), no concuerda con los estudios realizados en Australia y Malasia, donde esta variable ha fungido como predictor del automanejo en pacientes con ECNT ${ }^{12,14}$.

\section{Conclusiones}

Con base en los resultados se concluye que las variables predictoras identificadas en el presente estudio: autoeficacia, funcionalidad familiar, sintomatología de depresión, ejercicio y años con el diagnóstico, años de estudio y apoyo social percibido, representan importantes indicadores para entender las relaciones causa-efecto sobre el automanejo de personas con ECNT. Dicho conocimiento representa un aporte de suma relevancia, ya que no se detectaron estudios antecedentes que desarrollaran modelos predictivos del automanejo en México y Latinoamérica.

Asimismo, es importante destacar que los predictores antes mencionados confirman elementos importantes para el desarrollo de programas de automanejo, área que es promovida en el modelo de atención a crónicos de la OMS y en la actualidad es liderada por el área de Enfermería.

Con base en lo anterior, se puede concluir que el comportamiento de automanejo es complejo y no puede explicarse, ni predecirse, por un número limitado de variables. Por tal motivo, se sugiere continuar desarrollando modelos de trayectorias con base en los resultados del presente estudio y las teorías del comportamiento de automanejo. Del mismo modo se sugiere realizar este tipo de estudios con muestras probabilísticas y de diferentes poblaciones debido a las limitantes asociadas a la distribución atípica de las variables sexo, edad y tiempo de diagnóstico en esta investigación.

\section{Responsabilidades éticas}

Protección de personas y animales. Los autores declaran que en este estudio no se realizaron experimentaciones en seres humanos ni animales.

Confidencialidad de datos. Los autores declaran que en el artículo no aparecen datos de pacientes.

Derecho a la privacidad y consentimiento informado. Los autores declaran que han seguido los protocolos de su centro de trabajo sobre la publicación de datos de pacientes. Los autores han obtenido el consentimiento informado de los sujetos referidos en el artículo.

Financiamiento. Ninguno.

Conflicto de interés. Los autores declaran no tener conflictos de interés.

Este estudio se apegó a la Ley General de Salud en Materia de Investigación para la Salud y los principios éticos para la investigación médica en seres humanos de la Declaración de Helsinki, además el proyecto mayor de donde deriva este estudio fue avalado por el comité de ética de la Facultad de Enfermería Tampico de la Universidad Autónoma de Tamaulipas.

Los autores agradecen al Consejo Nacional de Ciencia y Tecnología y a la Secretaría de Salud de Tamaulipas. Jurisdicción No. 2 Tampico, por los apoyos y las facilidades otorgadas para llevar a cabo este estudio. 


\section{Referencias}

1. Organización Panamericana de la Salud. Cuidados innovadores para las condiciones crónicas: Organización y prestación de atención de alta calidad a las enfermedades crónicas no transmisibles en las Américas. Washington, DC: OPS 2013. [Consultado febrero 10 2017]. https://bit.ly/2CQ76Ty

2. Organización Mundial de la Salud. Informe sobre la situación mundial de las enfermedades no transmisibles 2014. Ginebra: OMS; 2014. [Consultado enero 10 2019]. Disponible en: https://bit.ly/2CFZO33

3. Organization for Economic Co-operation and Development and Pan American Health Organization. Applying modeling to improve health and economic policy decisions in the Americas: the case of non communicable diseases. Washington, DC: PAHO; 2015. [Consultado mayo 11 2017]. Disponible en: https://bit.ly/1SuXSd4

4. World Health Organization. Global report on diabetes. Geneva:WHO; 2016. [Consultado febrero 10 2017]. Disponible en: https://bit.ly/2UgwHNP

5. Instituto Nacional de Salud Pública. Encuesta Nacional de Salud y Nutrición de Medio Camino 2016. Mexico : INSP [Consultado febrero 10 2017]. Disponible en: https://bit.ly/2UgBxKZ

6. Forouzanfar MH, Afshin A, Alenxander LT, Anderson HR, Bhutta ZA, Biryukov S, et al. Global, regional, and national comparative risk assessmen of 79 behavioral, environmental and occupational, and metabolic risk or clusters of risk, 1990-2015: a systematic analysis for the Global Burder of Disease Study 2015. Lancet. 2016; 388(10053): 1659-1724. https://doi.org/10.1016/S0140-6736(16)31679-8

7. Instituto Mexicano para la Competitividad. Kilos de más, pesos de menos: los costos de la obesidad en México. México: IMCO; 2015. [Consultado mayo 11 2016]. Disponible en: https://bit.ly/1QQzMga

8. Lorig KR, Holman H. Self-management education: history, definition, outcomes and mechanisms. Ann Behav Med. 2003; 26(1): 1-7. https://doi.org/10.1207/S15324796ABM2601_01

9. Grady PA, Gough LL. Self-management: a comprehesive approach to management of chronic conditions. Am J Public Health. 2014; 104(8): 25-31. https://doi.org/10.2105/AJPH.2014.302041

10. Lawn S, Battersby M, Harvey P, Pols R, Ackland A. A behavioural therapy approach to self-management: the Flinders Program. Diabetes voice: bulletin of the International Diabetes Federation. 2009; 54(Special): 30-2.

11. Udlis KA. Self-management in chronic illness: concept and dimensional analysis. J Nurs Healthc Chronic Illn. 2011; 3(2): 130-9. https://doi.org/10.1111/j.1752-9824.2011.01085.x

12. Maneze D, Everett B, Astorga C, Yogendran D, Salamonson Y. The influence of health literacy and depression on diabetes self-management: a cross-sectional study. J Diabetes Res. 2016; 2016(3458969): 1-8. https://doi.org/10.1155/2016/3458969

13. Yadav S. Mental health problems and self-management among people with type-2 diabetes. IJHW. 2016; 7(10): 1021-3.

14. Gunggu A, Thon CC, Whye LC. Predictors of diabetes self-management among type 2 diabetes patients. J Diabetes Res. 2016; 2016(9158943): 7p. https://doi.org/10.1155/2016/9158943

15. Cousins R, Thomas C, Sharma D, Naderali EK. Sociodemographic and clinical predictors of selfmanagement among people with poorly controlled type 1 and type 2 diabetes: the role of illness perceptions and self-efficacy. J Diabetes Res. 2016; 12p. http://dx.doi.org/10.1155/2016/6708164

16. Rahimian-Boogar E, Besharat MA, Mohajeri-Tehrani M, Talepasand S. Predictive role of self-efficacy, belief of treatment effectiveness and social support in diabetes mellitus self-management. ijpcp. 2011; $17(3): 232-40$.

17. Peñarrieta-de Córdova I, Flores-Barrios F, Gutierrez-Gomes T, Piñonez-Martinez MS, Quintero-Valle LM, Castañeda-Hidalgo H. Self-management in chronic conditions: partners in health scale instrument validation. Nurs Manag. 2014; 20(10): 32-37. 
18. Peñarrieta MI, Flores-Barrios F, Gutiérrez-Gómez T, Piñones-Martínez S, Resendiz-Gonzalez E, Quintero-Valle LM. Self-management and family support in chronic diseases. J Nurs Educ Pract. 2015; 5(11): 73-80. http://dx.doi.org/10.5430/jnep.v5n11p73

19. Mar-García J, Peñarrieta-de Córdova M, León-Hernández R,Gutiérrez-Gómez T, Banda-González O, Rangel-Torres S, De León-Ramírez M. Relación entre automanejo y percepción de funcionalidad familiar en personas con diabetes mellitus tipo 2. Enferm. univ. 2017; 14(3): 155-61. http://dx.doi.org/10.1016/j.reu.2017.05.005

20. Martínez VY, Campbell SM, Hann M, Bower P. The individual contribution and relative importance of self-management and quality of care on glycaemic control in type 2 diabetes. Salud Publica Mex. 2016; 58(4): 404-11.

21. Peñarrieta-de Córdova MI, León R, Gutiérrez T, Mier N, Banda O, Delabra M. Effectiveness of a chronic disease self-management program in Mexico: a randomized controlled study. J Nurs Educ Pract. 2017; 7(7): 87-94. https://doi.org/10.5430/jnep.v7n7p87

22. Barceló A, Cafiero E, De Boer M, Escobar-Mesa A, García-López M, Jiménez RA, et al. Using collaborative learning to improve diabetes care and outcomes: The VIDA Project. Prim Care Diabetes. 2010; 4(3): 145-53. https://doi.org/10.1016/j.pcd.2010.04.005

23. Suarez-Cuba MA, Alcalá-Espinoza M. APGAR Familiar: una herramienta para detectar disfunción familiar. Revista Médica. 2014; $20(1)$ : 53-57.

24. Piña-López JL, Rivera-Icedo BM. Validación del cuestionario de apoyo social funcional en personas seropositivas al VIH del Noroeste de México. Cienc. enferm. 2007; XIII(2): 53-63. http://dx.doi.org/10.4067/S07 17-95532007000200007

25. Lorig K, Stewart A, Ritter P, Gonzalez V, Laurent D, Lynch J. Outcome measures for health education and other health care interventions. Thousand Oaks CA: Sage Publications; 1996. http://dx.doi.org/10.4135/9781452232966

26. Ritter PL, Ory MG, Laurent DD, Lorig K. Effects of chronic disease self-management programs for participants with higher depression scores: secondary analyses of an on-line and a small-group program. Transl Behav Med. 2014; 4(4): 398-406. https://doi.org/10.1007/s13142-014-0277-9

27. Arrieta J, Aguerrebere M, Raviola G, Flores H, Elliott P, Esponosa A, et al. Validity and utility of the patient health questionnaire (PHQ)-2 and PHQ-9 for screening and diagnosis of depression in rural Chiapas, Mexico: A cross-sectional study. J Clin Psychol. 2017; 73(9): 1076-90. https://doi.org/10.1002/jclp.22390

28. Lorig KL, Sobel DS, Ritter PL, Laurent D, Hobbs M. Effect of a self-management program on patients with chronic disease. Eff Clin Pract. 2001; 4(6): 256-62.

29. Battersby MW, Ask A, Reece MM, Markwick MJ, Collins JP.The Partners in Health Scale: the development and psychometric properties of a generic assessment scale for chronic condition self-management. Aust J Prim Health. 2003; 9(4): 41-52. https://doi.org/10.1071/PY03022 